\title{
Analysis of the near-band-edge luminescence of semiconductors containing isolated and bound shallow acceptors and donors
}

\author{
K.D. Glinchuk, A.V. Prokhorovich \\ Institute of Semiconductor Physics, NAS of Ukraine, 45 prospect Nauky, 03028 Kyiv, Ukraine
}

\begin{abstract}
Expressions for line intensities in the near-band-edge luminescence spectrum of semiconductors containing both isolated and bound shallow acceptors and donors are given. Found are the conditions when isolated and bound shallow acceptors and donors make rather small or dominating contributions into the near-band-edge luminescence spectrum. It is shown (on the basis of an analysis of the near-band-edge luminescence spectrum of semiinsulating $\mathrm{GaAs}$ ) that it is very probable for intensities of the near-band-edge luminescence lines to be determined by different states (isolated or bound) of shallow acceptors and donors in semiconductors of this type.
\end{abstract}

Keywords: semiconductors, luminescence, isolated acceptor, bound acceptor, shallow acceptor, shallow donor, GaAs.

Paper received 04.09 .02; revised manuscript received 15.11.02; accepted for publication 17.12.02.

\section{Introduction}

It is known that intensive near-band-edge emission lines are observed in semiconductor luminescence spectra (impurity and exciton-impurity lines; their appearance is caused by recombination of electrons and holes via shallow acceptors and donors as well as by annihilation of excitons bound with them). When calculating intensities of emission lines in the near-band-edge luminescence spectra, it is usually assumed that semiconductors contain isolated (see, for example, [1-4]), or bound in donor-acceptor pairs (see, for example, $[2,3,5,6]$ ), shallow acceptors and donors (in the following acceptors and donors). However, obviously, doped semiconductors contain both isolated and bound acceptors and donors. This work is devoted to a calculation of line intensities in the nearband-edge luminescence spectra of semiconductors containing isolated and bound acceptors and donors. We will show that, in a discussed case, the contribution of isolated and bound acceptors and donors into formation of the near-band-edge luminescence spectrum is not always determined by the relation between their concentrations. From this follows that it is very probable that in semiconductors isolated and bound acceptors and donors could make essentially different contributions into line intensities in the near-band-edge luminescence spectrum, even if their concentrations are approximately equal. Relations obtained will be used for elucidation of the relative role of isolated and bound acceptors and donors in the formation of the near-band-edge luminescence spectrum of semi-insulating gallium arsenide crystals.

\section{Model and main assumptions}

We will consider semiconductors at low temperatures [no thermally stimulated processes are observed in them, their conductivity is determined by uniformly distributed photoelectrons (concentration $\delta n$ ) and photoholes (concentration $\delta p$ )]. Let them contain $N_{A 1}$ and $N_{D 1}$ concentrations of isolated and $N_{A 2}$ and $N_{D 2}$ concentrations of bound acceptors and donors, accordingly (the total acceptor and donor concentrations are $N_{A}=N_{A 1}+N_{A 2}$ and $N_{D}=N_{D 1}+$ $+N_{D 2}$ ). The concentrations of neutral acceptors as well as ionized and neutral donors in the isolated state are $N_{A^{0} 1}, N_{D^{+} 1}, N_{D^{0} 1_{1}}$ and in the bound state are $N_{A^{0}{ }_{2}}$, $N_{D^{+} 2}, N_{D^{0}}$ (obviously, $N_{D^{+} 1}+N_{D^{0_{1}}}=N_{D 1}$ and $N_{D^{+}{ }_{2}}+N_{D^{0}}=N_{D 2}$ ). The capture coefficient of free electrons by neutral acceptors is $c_{n A}^{0}$ and by ionized do- 
nors is $c_{n D}^{+}$; the capture coefficient of free holes by ionized acceptors is $c_{p A}^{-}$and by neutral donors is $c_{p D}^{0}$. The average coefficient of inter-impurity recombination is $c_{n}^{*}$ [6]. The occupation probabilities of bound (at low tempo of inter-impurity transitions) and isolated acceptors by holes $\varphi_{A^{0}}$ and donors by electrons $\varphi_{D^{0}}$ and holes $1-\varphi_{D^{0}}$ are $\varphi_{A^{0}}=c_{p A}^{-} \delta p /\left(c_{p A}^{-} \delta p+c_{n A}^{0} \delta n\right)$,

$\varphi_{D^{0}}=c_{n D}^{+} \delta n /\left(c_{n D}^{+} \delta n+c_{p D}^{0} \delta p\right)$,

$1-\varphi_{D^{0}}=c_{p D}^{0} \delta p /\left(c_{n D}^{+} \delta n+c_{p D}^{0} \delta p\right)$

(see Appendix 1). Free electrons and holes could form (with the probability $b_{X}$ ) free excitons $X$ (their concentration is $n_{X}$, the probability of radiative annihilation is $\alpha_{X}$ ). Free excitons could be captured by neutral acceptors $A^{0}$ and ionized $D^{+}$and neutral donors $D^{0}$ (the capture coefficients are $b_{A^{0} X}, b_{D^{+} X}$ and $b_{D^{0} X}$, accordingly), forming exciton-impurity complexes $A^{0} X, D^{+} X$ and $D^{0} X$ (their concentrations are $n_{A^{0} X 1}, n_{D^{+} X 1}$, $n_{D^{0} X 1}$, if isolated acceptors and donors and $n_{A^{0} X 2}$, $n_{D^{+} X 2}, n_{D^{0} X 2}$, if bound acceptors and donors are components of $A^{0} X, D^{+} X$ and $D^{0} X$ complexes; the probabilities of radiative annihilation of bound excitons $A^{0} X, D^{+} X$ and $D^{0} X$ are $\alpha_{A^{0} X}, \alpha_{D^{+} X}$ and $\left.\alpha_{D^{0} X}\right)$.

Below, when writing the theoretical expressions for line intensities in the near-band-edge luminescence spectrum [induced by the free electron $(e)$ recombination on neutral acceptors $I_{e A^{0}}$, the free hole $(h)$ recombination on neutral donors $I_{h D^{0}}^{e A}$, the donor-acceptor recombination $I_{D^{0} A^{0}}$, annihilation of bound excitons $A^{0} X, D^{+} X$ and $D^{0} X$ accordingly $I_{A^{0} X}, I_{D^{+} X}$ and $I_{D^{0} X}$, see Fig. 1], we suppose that the mentioned recombination transitions are mainly radiative [6]. Besides that, expressions for line intensities in the near-band-edge luminescence spectra will be given for very probable relations between $c_{n D}^{+} N_{D 2} \delta n=b$ and $c_{p A}^{-} N_{A 2} \delta p=d(b>>d, b \approx d$ and $d>>b)$, at low $\left[c_{n}^{*}(b+d)>>\left(c_{n D}^{+} \delta n+c_{p D}^{0} \delta p\right)\left(c_{p A}^{-} \delta p+\right.\right.$ $\left.\left.+c_{n A}^{0} \delta n\right)=a\right]$ and high $\left[a>>c_{n}^{*}(b+d)\right]$ values of $\delta p$ and $\delta n$, and for low $n_{X}\left(b_{A^{0} X} n_{X}<<\alpha_{A^{0} X}, b_{D^{+} X} n_{X}<<\alpha_{D^{+} X}\right.$ and $\left.b_{D^{0} X} n_{X}<<\alpha_{D^{0} X}\right)$. This is connected with the fact that only in pointed cases convenient analytical expres-

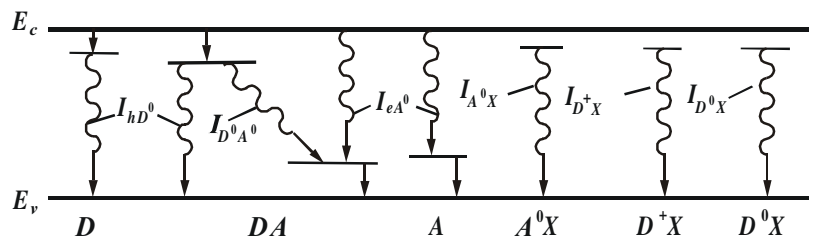

Fig.1. Radiative (wavy lines) and non-radiative ( continuous lines) transitions to isolated donors $\mathrm{D}$ and acceptors $\mathrm{A}$, in donoracceptor pairs DA and in bound excitons $A^{0} X, D^{+} X$ and $D^{0} X$. sions for the concentrations of bound acceptors and donors in neutral and ionized states and excitons captured by them are possible (see Appendixes 1 and 2).

\section{Theoretical relations for line intensities in the near-band-edge luminescence spectrum for semiconductors containing isolated and bound acceptors and donors}

\subsection{General relations}

Obviously, for the case, the discussed near-band-edge luminescence spectrum will be formed by electron-hole transitions to isolated and bound acceptors and donors and by annihilation of excitons captured by them (see Fig. 1). Undoubtedly, line intensities in the near-bandedge luminescence spectrum will be determined by the concentrations of neutral acceptors and ionized and neutral donors which are in isolated and bound states, and also by the concentrations of photoholes, photoelectrons and free excitons [it is possible that not only $\delta p$ and $\delta n$, but also $n_{X}$ (see Appendix 2) depend on $N_{A}$ and $N_{D}$ ]. They are equal (see Fig. 1):

$I_{e A^{0}}=c_{n A}^{0}\left(N_{A^{0} 1}+N_{A^{0}{ }_{2}}\right) \delta n$,

$I_{h D^{0}}=c_{p D}^{0}\left(N_{D^{0}}+N_{D^{0}}\right) \delta p$

$I_{D^{0} A^{0}}=c_{n}^{*} N_{D^{0}} N_{A^{0}}$,

$I_{A^{0} X}=b_{A^{0} X}\left(N_{A^{0}{ }_{1}}+N_{A^{0}{ }_{2}}\right) n_{X}$,

$I_{D^{+} X}=b_{D^{+} X}\left(N_{D^{+} 1}+N_{D^{+}{ }_{2}}\right) n_{X}$

$I_{D^{0} X}=b_{D^{0} X}\left(N_{D^{0}{ }_{1}}+N_{D^{0}{ }_{2}}\right) n_{X} \cdot$

When writing the Eqs (4)-(6) for $I_{A^{0} X}, I_{D^{+} X}$ and $I_{D^{0} X}$ values, we assumed that a small number of neutral acceptors as well as ionized and neutral donors could bind free excitons, i.e. $n_{A^{0} X 1,2} \ll N_{A^{0} 1,2}, n_{D^{+} X 1,2}<<N_{D^{+} 1,2}$ and $n_{D^{0}{ }_{X 1,2}}<N_{D^{0} 1,2}$ [this is valid at low $n_{X}$ values $\left(n_{X}<<\alpha_{A^{0} X} / b_{A^{0} X}, \alpha_{D^{+} X} / b_{D^{+} X}, \alpha_{D^{0} X} / b_{D^{0} X}\right)$, see Appendix 2].

In Eqs (1)-(6) (see Appendix 1):

$$
\begin{aligned}
& N_{A^{0} 1}=\varphi_{A^{0}} N_{A 1}, N_{D^{+} 1}=\left(1-\varphi_{D^{0}}\right) N_{D 1}, \\
& N_{D^{0} 1}=\varphi_{D^{0}} N_{D 1}
\end{aligned}
$$

at any $\delta p$ and $\delta n$ values;

$$
N_{A^{0} 2} \approx \frac{c_{p A}^{-} \delta p}{c_{n}^{*} \varphi_{D^{0}} N_{D 2}} N_{A 2}<<N_{A 2}
$$




$$
\begin{aligned}
& N_{D^{+} 2} \approx \frac{c_{p D}^{0} N_{D 2}+c_{p A}^{-} N_{A 2}}{c_{n D}^{+} \delta n+c_{p D}^{0} \delta p} \delta p, \\
& N_{D^{0} 2} \approx \frac{c_{n D}^{+} \delta n}{c_{n D}^{+} \delta n+c_{p D}^{0} \delta p} N_{D 2}
\end{aligned}
$$

at $b>>d$ and low $\delta p, \delta n$ values $\left(c_{n}^{*} b>>a\right)$ [then $c_{n}^{*} \varphi_{D^{0}} N_{D 2}>c_{p A}^{-} \delta p+c_{n A}^{0} \delta n$ and

$c_{p A}^{-} N_{A 2} \delta p /\left(c_{n D}^{+} \delta n+c_{p D}^{0} \delta p\right)<<N_{D 2}$; obviously, in this case, if $c_{p A}^{-} N_{A 2}>>c_{p D}^{0} N_{D 2}$, i.e.

$b>>c_{p D}^{0} N_{D 2} \delta p$,

then $c_{n D}^{+} \delta n>>c_{p D}^{0} \delta p$ and so, $\left.N_{D^{+} 2} \approx c_{p A}^{-} N_{A 2} \delta p / c_{n D}^{+} \delta n\right]$;

$N_{A^{0} 2} \approx \frac{c_{p A}^{-} \delta p}{c_{p A}^{-} \delta p+c_{n A}^{0} \delta n} N_{A 2}$,

$N_{D^{+} 2} \approx N_{D 2}$,

$N_{D^{0} 2} \approx \frac{c_{n D}^{+} \delta n}{c_{n}^{*} \varphi_{A^{0}} N_{A 2}} N_{D 2} \ll N_{D 2}$

at $d>>b$ and low $\delta p, \delta n$ values $\left(c_{n}^{*} d>>a\right)$ [then $\left.c_{n}^{*} \varphi_{A}{ }^{0} N_{A 2} \gg c_{n D}^{+} \delta n+c_{p D}^{0} \delta p\right]$;

$N_{A^{0}{ }_{2}} \approx\left(\frac{c_{n D}^{+} \delta n+c_{p D}^{0} \delta p}{c_{p A}^{-} \delta p+c_{n A}^{0} \delta n} \cdot \frac{c_{p A}^{-} \delta p}{c_{n}^{*} N_{A 2}}\right)^{1 / 2} N_{A 2} \ll N_{A 2}$,

$N_{D^{+} 2} \approx N_{D 2}$,

$N_{D^{0} 2} \approx\left(\frac{c_{p A}^{-} \delta p+c_{n A}^{0} \delta n}{c_{n D}^{+} \delta n+c_{p D}^{0} \delta p} \cdot \frac{c_{n D}^{+} \delta n}{c_{n}^{*} N_{D 2}}\right)^{1 / 2} N_{D 2} \ll N_{D 2}$

at $b \approx d\left(|b-d|<<a / c_{n}^{*}\right)$ and low $\delta p$ and $\delta n$ values $\left(c_{n}^{*} b\right.$, $\left.c_{n}^{*} d>>a\right)$

$N_{D^{+} 2} \approx \frac{c_{p D}^{0} \delta p}{c_{n D}^{+} \delta n+c_{p D}^{0} \delta p} N_{D 2}+\frac{\varphi_{A}^{0} \varphi_{D^{0}}^{2}}{c_{n D}^{+} \delta n} c_{n}^{*} N_{A 2} N_{D 2}$,

$N_{A^{0}}$ is given by the Eq. (10) and $N_{D^{0}}$ - by the Eq. (9) at any $b, d$ and high $\delta p, \delta n$ values $\left(a>>c_{n}^{*} b, c_{n}^{*} d\right)$ [obviously, $\varphi_{A} \varphi^{0}{ }_{D}^{2} c_{n}^{*} N_{A 2} N_{D 2} / c_{n D}^{+} \delta n \ll N_{D 2}$ in Eq. (13)];

$N_{D^{+} 2} \approx \frac{c_{p D}^{0} \delta p+c_{n}^{*} N_{A 2}}{c_{n D}^{+} \delta n} N_{D 2} \ll<N_{D 2}$

SQO, 5(4), 2002 at high $\delta p, \delta n$ values and $\varphi_{D^{0}} \approx \varphi_{A^{0}} \approx 1$ (i.e. $c_{n D}^{+} \delta n>>$ $c_{p D}^{0} \delta p$ and $\left.c_{p A}^{-} \delta p>>c_{n A}^{0} \delta n\right)$ [see Eq. (13)];

$N_{D^{+} 2} \approx \frac{c_{p D}^{0} \delta p}{c_{n D}^{+} \delta n+c_{p D}^{0} \delta p} N_{D 2}$

at high $\delta p, \delta n$ values and

$1-\varphi_{D^{0}} \gg \varphi_{A} \varphi^{0}{ }_{D}^{2} c_{n}^{*} N_{A 2} / c_{n D}^{+} \delta n$,

i.e. $c_{p D}^{0} \delta p>>\varphi_{A} \varphi_{D^{0}} c_{n}^{*} N_{A 2}$ [see Eq.(13)].

Further we consider that the $N_{D^{+}{ }_{2}}$ value at high $\delta p$ and $\delta n$ is given by the Eq. (14).

It follows from Eqs (1)-(6) that the relative role of isolated and bound acceptors and donors in determining intensities of the near-band-edge luminescence lines depends on the relation between $N_{A^{0}}$ and $N_{A^{0}}, N_{D^{+}}$ and $N_{D^{+}}, N_{D^{0}}$ and $N_{D^{0}}$ values (they are determined by isolated acceptors and donors if $N_{A^{0}{ }_{1}}>N_{A^{0}}$, $N_{D^{+}{ }_{1}} \gg N_{D^{+}{ }_{2}}$ and $N_{D^{0}{ }_{1}} \gg N_{D^{0}}$, as well as by bound acceptors and donors, if $N_{A^{0}{ }_{2}}>N_{A^{0}{ }_{1}}, N_{D^{+}{ }_{2}}>N_{D^{+}}$ and $N_{D^{0}} \gg N_{D^{0}}$ ). Besides that, one could also see: a) intensities $I_{e A^{0}}$ and $I_{A^{0} X}$ are determined by the same acceptor state (isolated, if $N_{A^{0} 0_{1}}>N_{A^{0}}$, and bound, if $N_{A^{0}{ }_{2}}>N_{A^{0} 1}$ ); b) intensities $I_{h D^{0}}$ and $I_{D^{0} X}$ are determined by the same donor state (isolated, if $N_{D^{0}}>>$ $N_{D^{0}}$, and bound, if $N_{D^{0}}>N_{D^{0}}$ ).

Equations (1)-(12) and (14) will be used below for determination at low and high photohole and photoelectron concentrations and low free exciton concentration of intensities of the near-edge-band luminescence lines in semiconductors containing both isolated and bound acceptors and donors. The obtained expressions for $I_{e A^{0}}$, $I_{h D^{0}}, I_{A^{0} X}, I_{D^{+} X}$ and $I_{D^{0} X}$ will be given in the form of two terms - the first one shows the contribution to intensities of the near-edge-band luminescence lines of isolated and the second one - of bound acceptors and donors.

\subsection{Low $\delta n$, $\delta p$ and $n_{X}$ values}

In this subsection when calculating luminescence line intensities, we consider that in a semiconductor the following relations between recombination characteristics $b$ and $d, c_{n D}^{+} \delta n$ and $c_{p D}^{0} \delta p, c_{p A}^{-} \delta p$ and $c_{n A}^{0} \delta n$ can realize: a) $b \gg>d, c_{n D}^{+} \delta n>c_{p D}^{0} \delta p$ and $c_{p A}^{-} \delta p>c_{n A}^{0} \delta n$ or $c_{p A}^{-} \delta p<<c_{n A}^{0} \delta n$

b) $d>>b, c_{p A}^{-} \delta p>>c_{n A}^{0} \delta n$ and $c_{n D}^{+} \delta n>c_{p D}^{0} \delta p$ or $c_{n D}^{+} \delta n<<c_{p D}^{0} \delta p$

c) $b \approx d, c_{n D}^{+} \delta n>>c_{p D}^{0} \delta p$ and $c_{p A}^{-} \delta p>>c_{n A}^{0} \delta n$. 
Undoubtedly, no problem exists in examination using Eqs (7)-(14) and (A5)-(A10) (see Appendix 1) for $N_{A^{0}}$, $N_{D^{+}}$and $N_{D^{0}}$ of any relations between $b$ and $d, c_{n D^{+}} \delta n$ and $c_{p D}^{0} \delta p, c_{p A}^{-} \delta p$ and $c_{n A}^{0} \delta n$ values.

a1) $c_{n D}^{+} N_{D 2} \delta n \gg c_{p A}^{-} N_{A 2} \delta p, c_{n D}^{+} \delta n \gg c_{p D}^{0} \delta p$ and $\boldsymbol{c}_{\boldsymbol{p} \boldsymbol{A}}^{-} \boldsymbol{\delta} \boldsymbol{p} \gg \boldsymbol{c}_{\boldsymbol{n} \boldsymbol{A}} \boldsymbol{\delta} \boldsymbol{n}$ (then $\varphi_{D^{0}} \approx \varphi_{A^{0}} \cong 1$ ). At the pointed relations between donor and acceptor recombination characteristics, it follows from Eqs. (7)-(9) that $N_{A^{0} 1} \cong N_{A 1}, N_{D^{+} 1} \cong\left(c_{p D}^{0} \delta p / c_{n D}^{+} \delta n\right) N_{D 1}<<N_{D^{0}} \cong N_{D 1}$ (i.e. isolated acceptors are filled by holes and isolated donors by electrons), $N_{A^{0}{ }_{2}} \cong\left(c_{p A}^{-} \delta p / c_{n}^{*} N_{D 2}\right) N_{A 2}<<N_{A 2}$ and $N_{D^{+} 2} \cong\left(c_{p D}^{0} N_{D 2}+c_{p A}^{-} N_{A 2}\right) \delta p / c_{n D}^{+} \delta n<<N_{D^{0}} \cong N_{D 2}$ (i.e. bound acceptors and donors are filled by electrons).

So, if above given inequalities are valid, the discussed intensities of impurity, inter-impurity and excitonic luminescence lines, as follows from Eqs.(1)-(9), are given by the following equations [here, it is obviously, $c_{p A}^{-} \delta p / c_{n}^{*} N_{D 2}, \quad c_{p D}^{0} \delta p / c_{n D}^{+} \delta n<1,\left(c_{p D}^{0} N_{D 2}+\right.$ $\left.+c_{p A}^{-} N_{A 2}\right) \delta p<<c_{n D}^{+} N_{D 2} \delta n$ and $\left.N_{D 1}+N_{D 2}=N_{D}\right]$ :

$$
\begin{aligned}
& I_{e A^{0}}=c_{n A^{0}}^{0}\left(N_{A 1}+\frac{c_{p A}^{-} \delta p}{c_{n}^{*} N_{D 2}} N_{A 2}\right) \delta n \\
& I_{h D^{0}}=c_{p D}^{0}\left(N_{D 1}+N_{D 2}\right) \delta p \\
& I_{D^{0} A^{0}}=c_{p A^{-}}^{-} N_{A 2} \delta p \\
& I_{A^{0} X}=b_{A^{0} X}\left(N_{A 1}+\frac{c_{p A}^{-} \delta p}{c_{n}^{*} N_{D 2}} N_{A 2}\right) n_{X}, \\
& I_{D^{+} X}=b_{D^{+} X} \frac{\delta p}{c_{n D}^{+} \delta n}\left[c_{p D^{0}}^{0} N_{D 1}+\left(c_{p D}^{0} N_{D 2}+c_{p A^{-}}^{-} N_{A 2}\right)\right] n_{X}, \\
& I_{D^{0} X}=b_{D^{0} X}\left(N_{D 1}+N_{D 2}\right) n_{X} .
\end{aligned}
$$

In this case: (1) $I_{e A^{0}} / I_{D^{0} A^{0}}=c_{n A}^{0} N_{A 1} \delta n / c_{p A}^{-} N_{A 2} \delta p$, if $I_{e A^{0}}$ is determined by isolated acceptors (then $I_{e A^{0}} \ll I_{D^{0} A^{0}}$, if $\left.N_{A 2} \geq N_{A 1}\right)$, and $I_{e A^{0}} / I_{D^{0} A^{0}}=$ $=c_{n A}^{0} \delta n / c_{n}^{*} N_{D 2}<<1$, if $I_{e A^{0}}$ is determined by bound acceptors; (2) $I_{h D^{0}} / I_{D^{0} A^{0}}=c_{p D^{0}}^{0} N_{D 1} / c_{p A^{-}}^{-} N_{A 2}$, if $I_{h D^{0}}$ is determined by isolated donors, and $I_{h D^{0}} / I_{D^{0} A^{0}}=$ $=c_{p D}^{0} N_{D 2} / c_{p A}^{-} N_{A 2}$, if $I_{h D^{0}}$ is determined by bound donors (it is very probable that $I_{h D^{0}}<I_{D^{0} A^{0}}$ as one expects that $\left.c_{p D}^{0} \ll c_{p A}^{-}[4]\right) ;(3) I_{e A^{0}} \sim \delta$ and $I_{A^{0} X} \sim n_{X}$, if the corresponding emission is caused by isolated acceptors, and $I_{e A^{0}} \sim \delta p \delta n$ and $I_{A^{0} X} \sim n_{X} \delta p$, if luminescence is caused by bound acceptors; (4) $I_{h D^{0}} \sim \delta p, I_{D^{+} X} \sim(\delta p /$ $\delta$ ) $n_{X}$ and $I_{D^{0}} \sim n_{X}$ if luminescence is induced by isolated or bound donors.

a2) $c_{n D}^{+} N_{D 2} \delta n \gg c_{p A}^{-} N_{A 2} \delta p, c_{n D}^{+} \delta n \gg c_{p D}^{0} \delta p$ and $\boldsymbol{c}_{\boldsymbol{p} \boldsymbol{A}} \boldsymbol{\delta} \boldsymbol{p} \ll \boldsymbol{c}_{\boldsymbol{n} \boldsymbol{A}}^{\mathbf{\delta}} \boldsymbol{\delta} \boldsymbol{n}$ (then $\varphi_{D^{0}} \cong 1$ and $\varphi_{A^{0}} \cong$ $\left.\cong c_{p A}^{-} \delta p / c_{n A}^{0} \delta n<<1\right)$. At the pointed relations between donor and acceptor recombination characteristics, as it follows from Eqs. (7)-(9), $N_{D^{+}{ }_{1}}, N_{D^{0}{ }_{1}}, N_{A^{0}}, N_{D^{+} 2}$ and $N_{D^{0}}$ values and occupations of isolated donors as well as bound acceptors and donors by holes and electrons are the same as given in the subsection 3.2.a1, and $N_{A^{0} 1} \cong\left(c_{p A}^{-} \delta p / c_{n A}^{0} \delta n\right) N_{A 1} \ll N_{A 1}$ (i.e. isolated acceptors are filled by electrons). Undoubtedly, here the intensities $I_{h D^{0}}, I_{D^{0} A^{0}}, I_{D^{+} X}$ and $I_{D^{0} X}$ are determined, accordingly, by Eqs (16), (17), (19) and (20), and

$$
\begin{aligned}
& I_{e A^{0}}=c_{p A}^{-}\left(N_{A 1}+\frac{c_{n A}^{0} \delta n}{c_{n}^{*} N_{D 2}} N_{A 2}\right) \delta p, \\
& I_{A^{0} X}=b_{A^{0} X} \frac{c_{p A}^{-} \delta p}{c_{n A}^{0} \delta n}\left(N_{A 1}+\frac{c_{n A}^{0} \delta n}{c_{n}^{*} N_{D 2}} N_{A 2}\right) n_{X},
\end{aligned}
$$

where $c_{n A}^{0} \delta n / c_{n}^{*} N_{D 2}, c_{p A}^{-} \delta p / c_{n A}^{0} \delta n<<1$.

In this case, in contrast to examined in the subsection 3.2. a1, $I_{e A^{0}} / I_{D^{0} A^{0}}=N_{A 1} / N_{A 2}$ (then $I_{e A^{0}}>I_{D^{0} A^{0}}$, if $N_{A 1}>N_{A 2}$, and $I_{e A^{0}}<I_{D^{0} A^{0}}$, if $\left.N_{A 1}<N_{A 2}\right)$, and $I_{e A^{0}} \sim \delta p$ and $I_{A^{0} X} \sim(\delta p / \delta n) n_{X}$, if $I_{e A^{0}}$ and $I_{A^{0} X}$ are induced by isolated acceptors.

b1) $c_{p A}^{-} N_{A 2} \delta p \gg c_{n D}^{+} N_{D 2} \delta n, c_{p A}^{-} \delta p \gg c_{n A}^{0} \delta n$ and $c_{n D}^{+} \delta n \gg c_{p D}^{0} \delta p$ (then $\varphi_{A} \approx \approx \varphi_{D^{0}} \cong 1$ ). If these inequalities are valid, then it follows from Eqs (7), (10) and (11) that $N_{A^{0} 1} \cong N_{A 1}, N_{D^{+} 1}=\left(c_{p D}^{0} \delta p / c_{n D}^{+} \delta n\right) N_{D 1}<<$ $N_{D^{0}} \cong N_{D 1}$ (i.e. isolated acceptors are filled by holes and isolated donors - by electrons), $N_{A^{0}{ }_{2}} \cong N_{A 2}$ and $N_{D^{+} 2} \cong N_{D 2}>N_{D^{0}} \cong\left(c_{n D}^{+} \delta n / c_{n}^{*} N_{A 2}\right) N_{D 2}$ (i.e. bound acceptors and donors are filled by holes). Undoubtedly, if the above inequalities are valid, then the nearband-edge luminescence intensities, as follows from Eqs (1)-(7), (10) and (11), are determined by the following expressions $\left(c_{n D}^{+} \delta n / c_{n}^{*} N_{A 2}, c_{p D}^{0} \delta p / c_{n D}^{+} \delta n<<1\right.$ and $N_{A 1}+N_{A 2}=N_{A}$ in them) .

$$
I_{e A^{0}}=c_{n A}^{0}\left(N_{A 1}+N_{A 2}\right) \delta n
$$

$I_{h D^{0}}=c_{p D}^{0}\left(N_{D 1}+\frac{c_{n D}^{+} \delta n}{c_{n}^{*} N_{A 2}} N_{D 2}\right) \delta p$,

$I_{D^{0} A^{0}}=c_{n D}^{+} N_{D 2} \delta n$,

$$
I_{A^{0} X}=b_{A^{0} X}\left(N_{A 1}+N_{A 2}\right) n_{X}
$$




$$
\begin{aligned}
& I_{D^{+} X}=b_{D^{+} X}\left(\frac{c_{p D}^{0} \delta p}{c_{n D}^{+} \delta n} N_{D 1}+N_{D 2}\right) n_{X}, \\
& I_{D^{0} X}=b_{D^{0} X}\left(N_{D 1}+\frac{c_{n D}^{+} \delta n}{c_{n}^{*} N_{A 2}} N_{D 2}\right) n_{X} .
\end{aligned}
$$

In conditions considered: (1) $I_{e A^{0}} / I_{D^{0} A^{0}}=c_{n A}^{0} N_{A 1} /$ $c_{n D}^{+} N_{D 2}$, if $I_{e A^{0}}$ is determined by isolated acceptors, and $I_{e A^{0}} / I_{D^{0} A^{0}} \stackrel{e A}{=} c_{n A}^{0} N_{A 2} / c_{n D}^{+} N_{D 2}$, if $I_{e A^{0}}$ is determined by bound acceptors (it is very probable that $I_{e A^{0}}<<I_{D^{0} A^{0}}$, as one expects that $c_{n D}^{+} \gg c_{n A}^{0}$ [4]); (2) $I_{h D^{0}} / I_{D^{0} A^{0}}=$ $c_{p D}^{0} N_{D 1} \delta p / c_{n D}^{+} N_{D 2} \delta n$, if $I_{h D^{0}}$ is determined by isolated donors (then, obviously, $I_{h D^{0}}<<I_{D^{0} A^{0}}$, if $N_{D 2} \geq N_{D 1}$ ), and $I_{h D^{0}} / I_{D^{0} A^{0}}=c_{p D}^{0} \delta p / c_{n}^{*} N_{A 2}<<1$, if $I_{h D^{0}}$ is determined by bound donors; (3) $I_{e A^{0}} \sim \delta n$ and $I_{A^{0} X} \sim n_{X}$, if luminescence is caused by isolated or bound acceptors; (4) $I_{h D^{0}} \sim \delta p, I_{D^{+} X} \sim(\delta p / \delta n) n_{X}$ and $I_{D^{0} X} \sim n_{X}$, if the discussed luminescence is induced by isolated donors, and $I_{h D^{0}} \sim \delta n \delta p, I_{D^{+} X} \sim n_{X}$ and $I_{D^{0} X} \sim n_{X} \delta n$, if it is caused by bound donors.

b2) $c_{p A}^{-} N_{A 2} \delta p \gg c_{n D}^{+} N_{D 2} \delta n, c_{p A}^{-} \delta p \gg c_{n A}^{0} \delta n$ and $\boldsymbol{c}_{\boldsymbol{n} D}^{+} \delta \boldsymbol{n} \ll \boldsymbol{c}_{\boldsymbol{p} D}^{\mathbf{0}} \delta \boldsymbol{p}$ (then $\varphi_{A^{0}} \cong 1$ and $\varphi_{D^{0}} \cong c_{n D^{+}}^{+} \delta n /$ $\left.c_{p D}^{0} \delta p<<1\right)$. At the pointed relations between the acceptor and donor recombination characteristics, as follows from Eqs (7), (10) and(11), $N_{A^{0} 1}, N_{A^{0}}, N_{D^{+} 2}$ and $N_{D^{0} 2}$ values and occupations of isolated acceptors as well as bound acceptors and donors by holes are the same as given in the subsection 3.2.b1, and $N_{D^{+}} \cong N_{D 1}>N_{D^{0}} \cong$ $\cong\left(c_{n D}^{+} \delta n / c_{p D}^{0} \delta p\right) N_{D 1}$ (i.e. isolated donors are filled by holes). In this case, the intensities $I_{e A^{0}}, I_{D^{0} A^{0}}$ and $I_{A^{0} X}$ are determined accordingly to Eqs (23), (25) and (26), and

$$
\begin{aligned}
& I_{h D^{0}}=c_{n D}^{+}\left(N_{D 1}+\frac{c_{p D}^{0} \delta p}{c_{n}^{*} N_{A 2}} N_{D 2}\right) \delta n, \\
& I_{D^{+} X}=b_{D^{+} X}\left(N_{D 1}+N_{D 2}\right) n_{X}, \\
& I_{D^{0} X}=b_{D^{0} X} \frac{c_{n D}^{+} \delta n}{c_{p D}^{0} \delta p}\left(N_{D 1}+\frac{c_{p D}^{0} \delta p}{c_{n}^{*} N_{A 2}} N_{D 2}\right) n_{X},
\end{aligned}
$$

where $c_{p D}^{0} \delta p / c_{n}^{*} N_{A 2}, c_{n D}^{+} \delta n / c_{p D}^{0} \delta p<<1$ and $N_{D 1}+$ $+N_{D 2}=N_{D}$.

Obviously, here in contrast to examined in the subsection $3.2 \mathrm{~b} 1, I_{h D^{0}} / I_{D^{0} A^{0}}=N_{D 1} / N_{D 2}$ (then $I_{h D^{0}}>$ $I_{D^{0} A^{0}}$, if $N_{D 1}>N_{D 2}$, and $I_{h D^{0}}<I_{D^{0} A^{0}}$, if $\left.N_{D 1}<N_{D 2}\right)$, and $I_{h D^{0}} \sim \delta n, I_{D^{+} X} \sim n_{X}$ and $I_{D^{0} X} \sim(\delta n / \delta p) n_{X}$, if $I_{h D^{0}}, I_{D^{+} X}$ and $I_{D^{0} X}$ are determined by isolated donors. c2) $c_{n D}^{+} N_{D 2} \delta n \approx c_{p A}^{-} N_{A 2} \delta p, c_{n D}^{+} \delta n>c_{p D}^{0} \delta p$ and $\boldsymbol{c}_{\boldsymbol{p} \boldsymbol{A}}^{-} \boldsymbol{\delta} \boldsymbol{p} \gg \boldsymbol{c}_{\boldsymbol{n} \boldsymbol{A}}^{\mathbf{0}} \boldsymbol{\delta} \boldsymbol{n}$. In this case, as follows from Eqs (7) and (12), $N_{A^{0} 1} \approx N_{A 1}, N_{D^{+} 1} \approx\left(c_{p D}^{0} \delta p / c_{n D}^{+} \delta n\right) N_{D 1}<<$ $N_{D^{0}} \approx N_{D 1}$ and $N_{A^{0}} \approx\left(c_{n D^{+}} N_{A 2} \delta n / c_{n}^{*}\right)^{1 / 2}<<N_{A 2}$,

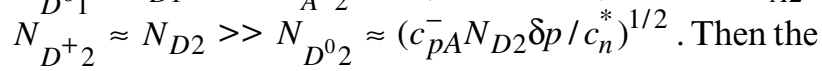
near-band-edge luminescence intensities are [see Eqs (1)(6)]:

$$
\begin{aligned}
& I_{e A^{0}}=c_{n A}^{0}\left(N_{A 1}+\sqrt{\frac{c_{n D}^{+} \delta n}{c_{n}^{*}} N_{A 2}}\right) \delta n, \\
& I_{h D^{0}}=c_{p D}^{0}\left(N_{D 1}+\sqrt{\frac{c_{p A}^{-} \delta p}{c_{n}^{*}} N_{D 2}}\right) \delta p, \\
& I_{A^{0} X}=b_{A^{0} X}\left(N_{A 1}+\sqrt{\frac{c_{n D^{*} \delta n}^{*} c_{n}^{*}}{c_{n}}}\right) n_{X}, \\
& I_{D^{0} X}=b_{D^{0} X}\left(N_{D 1}+\sqrt{\frac{c_{p A}^{-} \delta p}{c_{n}^{*}} N_{D 2}}\right) n_{X},
\end{aligned}
$$

$I_{D^{0} A^{0}}$ is given by the Eqs (17) and (25) (obviously, $I_{D^{0} A^{0}}>>$ $\left.I_{e A^{0}}, I_{h D^{0}}\right)$, and $I_{D^{+} X}$ - by the Eq (27). Evidently, $\left(c_{n D}^{+} N_{A 2} \delta n / c_{n}^{*}\right)^{1 / 2}<<N_{A 2}$ and $\left(c_{p A}^{-} N_{D 2} \delta p / c_{n}^{*}\right)^{1 / 2}<<$ $N_{D 2}$ in Eqs (32) and (33). Besides that: (1) $I_{e A} 0 \sim \delta n$, $I_{h D^{0}} \sim \delta p, I_{A^{0} X} \sim n_{X}, I_{D^{+} X} \sim(\delta p / \delta n) n_{X}$ and $I_{D^{0} X} \sim n_{X}$, if luminescence is caused by isolated acceptors or donors; (2) $I_{e A^{0}} \sim(\delta n)^{3 / 2}, I_{h D^{0}} \sim(\delta p)^{3 / 2}, I_{A^{0} X} \sim(\delta n)^{1 / 2} n_{X}$ and $I_{D^{0} X} \sim(\delta p)^{1 / 2} n_{X}$, if luminescence is caused by bound acceptors or donors.

\subsection{High $\delta n, \delta p$ and low $n_{X}$ values}

Undoubtedly, at high $\delta p, \delta n$ and low $n_{X}$ line intensities in the near-band-edge luminescence spectrum are (see Sec. 3.1):

$I_{e A^{0}}=c_{n A}^{0} \varphi_{A^{0}}\left(N_{A 1}+N_{A 2}\right) \delta n$,

$I_{h D^{0}}=c_{p D}^{0} \varphi_{D^{0}}\left(N_{D 1}+N_{D 2}\right) \delta p$,

$I_{D^{0} A^{0}}=c_{n}^{*} N_{A 2} N_{D 2}$,

$I_{A^{0} X}=b_{A^{0} X} \varphi_{A^{0}}\left(N_{A 1}+N_{A 2}\right) n_{X}$,

$I_{D^{+} X}=b_{D^{+} X}\left(1-\varphi_{D^{0}}\right)\left(N_{D 1}+N_{D 2}\right) n_{X}$,

$I_{D^{0} X}=b_{D^{0} X} \varphi_{D^{0}}\left(N_{D 1}+N_{D 2}\right) n_{X}$,

where, as was pointed above, $N_{A 1}+N_{A 2}=N_{A}$ and $N_{D 1}+N_{D 2}=N_{D}$. 
In this case : (1) $I_{e A^{0}}, I_{h D^{0}}>I_{D^{0} A^{0}} ;(2)$ the $I_{e A^{0}}$, $I_{h D^{0}}, I_{A^{0} X}, I_{D^{+} X}$ and $I_{D^{0} X}$ intensities change similarly with $\delta p$ and $\delta n$ variations independently of the isolated or bound acceptors and donors that are involved in the corresponding emissions.

\subsection{Discussion}

\subsubsection{Low $\delta n$, $\delta p$ and $n_{X}$ values}

In the above given equations for line intensities in nearband-edge luminescence spectrum at low concentrations of photoholes, photoelectrons and free excitons the following deserves special attention. Only in some cases [obviously, they are possible when $b>>d$ or $d>>b$ and are not possible when $b \approx d$, see Eqs (32) and (33)] the contribution of isolated and bound acceptors and donors into formation of the near-band-edge luminescence spectrum, i.e. in the corresponding emission intensities, depends only on the relation between their concentrations (it is proportional to their concentrations, if $\delta p$, $\delta n$ and $n_{X}$ do not depend on $N_{A}$ and $N_{D}$ ) - the near-edge-band luminescence spectrum is determined by isolated acceptors and donors, if $N_{A 1} \gg N_{A 2}$ and $N_{D 1} \gg N_{D 2}$, and, on the contrary, by bound acceptors and donors, if $N_{A 2}>>N_{A 1}$ and $N_{D 2}>>N_{D 1}$. So, if $b>>d$ the pointed refers to intensities $I_{h D^{0}}$ и $I_{D^{0} X}$, and if $d>>b$ it refers to intensities $I_{e A^{0}}, I_{A^{0} X}$ and $I_{D^{+} X}$ [see Eqs (16), (20), (23), (26) and (30)]. But in most cases the contribution of isolated and bound acceptors and donors into formation of the nearband-edge luminescence spectrum, i.e. in the corresponding emission intensities, depends not only on the relation between their concentrations but on their recombination characteristics too. So, at $N_{A 1}>>\left(c_{p A}^{-} \delta p / c_{n}^{*} N_{D 2}\right) N_{A 2}$, $\left(c_{n A}^{0} \delta n / c_{n}^{*} N_{D 2}\right) N_{A 2}$ and $N_{D 1}>\left(c_{n D}^{+} \delta n / c_{n}^{*} N_{A 2}\right) N_{D 2}$, $\left(c_{p D}^{0} \delta p / c_{n}^{*} N_{A 2}\right) N_{D 2}$, or at $N_{A 1}>\left(c_{n D}^{+} N_{A 2} \delta n / c_{n}^{*}\right)^{1 / 2}$ and $N_{D 1}>\left(c_{p A}^{-} N_{D 2} \delta p / c_{n}^{*}\right)^{1 / 2}$ the emission intensities $I_{e A^{0}}$ and $I_{A^{0} X}$ (if $\left.b>>d\right), I_{h D^{0}}$ and $I_{D^{0} X}$ (if $\left.d>>b\right)$, $I_{e A^{0}}, I_{A^{0} X}, I_{h D^{0}}$ and $I_{D^{0} X}$ (if $b \approx d$ ) are determined by isolated acceptors and donors, even when their concentrations $N_{A 1}$ and $N_{D 1}$ are smaller than the concentrations of bound acceptors $N_{A 2}$ and donors $N_{D 2}$ [see Eqs (15), (18), (21), (22), (24), (28), (29) and (31)-(33)]. At the same time, the excitonic emission intensity $I_{D^{+}}{ }_{X}$ can be determined : i) by bound acceptors (if $b>>d$ ), even if their concentration $N_{A 2}$ is essentially lower than the donor concentration $N_{D}$ [it takes place at $c_{p A}^{-} N_{A 2}>>c_{p D}^{0} N_{D}$, see Eq. (19)]; ii) by bound donors (at $d \approx b$ or $d>>b$ ), even if their concentration $N_{D 2}$ is essentially lower than the concentration of isolated donors $N_{D 1}$ [this takes place, if $N_{D 2}>>\left(c_{p D}^{0} \delta p / c_{n D}^{+} \delta n\right) N_{D 1}$, see Eq. (27) and Sec. 3.2 c]. Obviously, the intensity of interimpurity luminescence is determined only by bound acceptors and donors [see Eqs (17) and (25)].
In discussed conditions, it is very probable that $I_{e A^{0}}$, $I_{h D^{0}} \ll I_{D^{0} A^{0}}$ (see Sec. 3.2, a1, b1 and c). Only in specific cases (at $b>>d$ or $d>>b$ ) if $I_{e A^{0}}$ and $I_{h D^{0}}$, emission intensities are determined by isolated acceptors and donors as well as $N_{A 1}>N_{A 2}$ and $N_{D 1}>N_{D 2}$, it is possible that $I_{e A^{0}}, I_{h D^{0}}>I_{D^{0} A^{0}}$ (see Sec. 3.2, a 2 and b2).

\subsubsection{High $\delta n, \delta p$ and low $n_{X}$ values}

Undoubtedly, at high $\delta n, \delta p$ and low $n_{X}$ the contribution of isolated and bound acceptors and donors into formation of the near-band-edge luminescence spectrum, i.e. in the intensities of the impurity and exciton-impurity luminescence lines depends only on the relation between their concentrations (it is proportional to their concentrations, if $\delta n, \delta p$ and $n_{X}$ do not depend on $N_{A}$ and $N_{D}$ ) [see Eqs (34), (35) and (37)-(39)]. Obviously, in this case, as in a case of low $\delta p$ and $\delta n$ values, the intensity of the donor-acceptor luminescence is determined only by bound acceptors and donors [see Eq. (35)].

\section{An example: analysis of the near-band-edge luminescence spectrum of semi-insulating GaAs crystals}

\subsection{Experimental}

Luminescence was excited by a He-Ne laser (the wavelength $\lambda=632.8 \mathrm{~nm}$, the quantum energy $h v_{e}=1.96 \mathrm{eV}$, the intensity $L$ ). Conductivity of semi-insulating GaAs was determined by excess holes and electrons. In crystals investigated, the $\delta p$ and $\delta n$ values increased linearly ( $\delta p$, $\delta n \sim L)$ and the $n_{X}$ value - quadratically $\left(n_{X} \sim L^{2}\right)$ with the excitation intensity (the $n_{X}$ variations with $L$ were found from the excitation dependence of the emission intensity induced by the free exciton annihilation $I_{X}=$ $\left.=\alpha_{X} n_{X} \sim L^{2}[4]\right)$. At excitations used: a) the $\delta n, \delta p$ and b) $n_{X}$ values were low. The first (a) is confirmed by the observed excitation dependence of $I_{D^{0} A^{0}}-I_{D^{0}} A^{0}$ variations were proportional to the concentration of photocarriers [see Eqs (17) and (25)]. The second (b) follows from the observed excitation dependencies of $I_{A^{0} X}$, $I_{D^{+} X}, I_{D^{0} X}$ and $n_{X}-I_{A^{0} X}, I_{D^{+} X}, I_{D^{0} X}, n_{X} \sim \stackrel{A}{L}^{2}$, so $I_{A^{0} X}, I_{D^{+} X}, I_{D^{0} X} \sim n_{X}$ (see Appendix 2).

\subsection{Analysis of photoluminescence results}

Fig. 2 shows the $4.2 \mathrm{~K}$ near-band-edge luminescence spectrum and also the $4.2 \mathrm{~K}$ dependencies of line intensities in it on the excitation intensity $L$ for undoped semi-insulating gallium arsenide. Its form and line intensities are determined by: a) radiative transitions in bound acceptors and donors [the emission line $I_{D^{0} A^{0}}\left(I_{D^{0} A^{0}} \sim L\right)$ is induced]; b) radiative recombination of free electrons on isolated acceptors [the emission line $I_{e A^{0}}\left(I_{e A^{0}} \sim L\right)$ is SQO, 5(4), 2002 


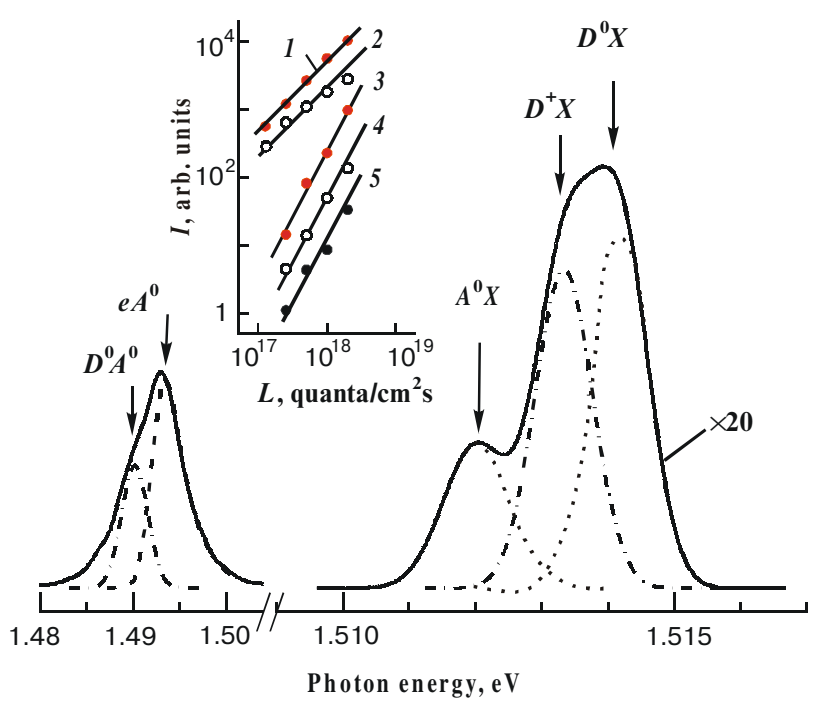

Fig.2. Near-band-edge luminescence spectrum of semi-insulating gallium arsenide (dotted curves are the result of a spectrum decomposition on individual components; the attribution of the spectral lines to transitions involving shallow acceptors and donors and excitons is indicated). In the inset - dependencies of intensities of the near-band-edge luminescence lines $I_{D^{0} A^{0}}$ (1), $I_{e A^{0}}$ (2), $I_{A^{0} X}$ (3), $I_{D^{+} X}$ (4) and $I_{D^{0} X}$ (5) on the excitation intensity (the relations between the line intensities are arbitrary; the true relations are seen from the spectrum). Measurements were conducted at low $\delta n, \delta p$ and $n_{X}$ values and $T=4.2 \mathrm{~K}$; the spectrum was recorded at $L=10^{18}$ quanta $/\left(\mathrm{cm}^{2} \cdot \mathrm{s}\right)$.

induced], and radiative annihilation of excitons bound with them (the emission line $I_{A^{0} X}$ is induced); c) radiative annihilation of excitons bound with isolated donors (the emission lines $I_{D^{+} X}$ and $I_{D^{0} X}$ are induced). Dominating role of isolated acceptors and donors in determining the intensities $I_{A^{0} X}, I_{D^{+} X}$ and $I_{D^{0} X}$ is confirmed by similar dependencies of intensities $I_{A^{0} X}, I_{D^{+} X}$ and $I_{D^{0} X}$ on $L$, namely: $I_{A^{0} X}, I_{D^{+} X}, I_{D^{0} X} \sim L^{2}$ (see Sec. 3.4.1). Dominating role of isolated acceptors in determining the $I_{e A^{0}}$ value is confirmed by the found defect nature (isolated acceptors), which is responsible for the $I_{A^{0} X}$ emission (as was noted in Sec. 3.1, the $I_{e A^{0}}$ and $I_{A^{0} X}$ emission intensities are induced by the same acceptor state). Besides that, this is also confirmed by nearly the same $I_{e A^{0}}$ and $I_{D^{0} A^{0}}$ values at any $L\left(I_{e A^{0}}, I_{D^{0} A^{0}} \sim\right.$ $L$, i.e. $I_{e A^{0}} / I_{D^{0} A^{0}}$ does not depend on $L$ ) (see Sec. 3.4.1). So, both isolated and bound acceptors and donors take part in the formation of the near-band edge luminescence spectrum of semi-insulating GaAs.

\section{Conclusion}

A contribution of isolated as well as bound acceptors and donors into intensities of the near-band-edge lumines-

SQO, 5(4), 2002 cence lines depends not only on the relation between their concentrations, but also on their recombination characteristics and concentrations of photoelectrons and photoholes in semiconductor. This fact (it is connected with the different role of inter-impurity transitions in determining the occupation of acceptors and donors by electrons and holes, i.e. the $N_{A^{0}}, N_{D^{+}}$and $N_{D^{0}}$ values [6]), must be taken into account, in particular, when the shallow acceptor and donor content in semiconductors is determined from the analysis of the near-band-edge luminescence spectra $[4,6]$

\section{Appendix}

\section{Equations for $N_{A^{0} 1,2}, \quad N_{D^{+}{ }_{1,2}}$ and $N_{D^{0}{ }_{1,2}}$}

For the model used and $L>0$ (see Fig. 1 and Sec. 2 ) the rate equations for $N_{A^{0} 1,2}, N_{D^{+} 1,2}$ and $N_{D^{0}{ }_{1,2}}$ are given by the following obvious expressions:

$\frac{d N_{A^{0} 1}}{d t}=c_{p A}^{-}\left(N_{A 1}-N_{A^{0} 1}\right) \delta p-c_{n A^{0}}^{0} N_{A^{0} 1} \delta n$,

$\frac{d N_{D^{0}}}{d t}=-\frac{d N_{D^{+}}}{d t}=c_{n D}^{+}\left(N_{D 1}-N_{D^{0} 1}\right) \delta n-c_{p D^{N} N_{D^{0}} \delta} \delta p$

$\frac{d N_{A^{0}}}{d t}=$

$=c_{p A}^{-}\left(N_{A 2}-N_{A^{0}{ }_{2}}\right) \delta p-c_{n A^{0}}^{0} N_{A^{0}{ }_{2}} \delta n-c_{n}^{*} N_{D^{0}{ }_{2}} N_{A^{0}{ }_{2}}$,

$\frac{d N_{D^{0}}}{d t}=-\frac{d N_{D^{+}}}{d t}=$

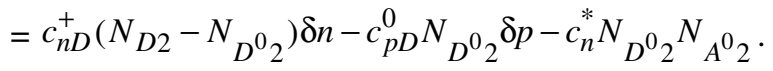

When writing the rate equations (A1)-(A4), it is implicitly assumed that the bound excitons recombine leaving neutral acceptors as well as ionized and neutral donors $[1,5]$. So the processes of annihilation of bound excitons do not influence the $N_{A^{0}}, N_{D^{+}}$and $N_{D^{0}}$ values, i.e. $N_{A^{0}}, N_{D^{+}}, N_{D^{0}} \neq f\left(n_{A^{0} X}, n_{D^{+} X}, n_{D^{0} X}\right)$.

In the steadystate $\left(d N_{A^{0}} / d t=d N_{D^{+}} / d t=d N_{D^{0}} / d t=\right.$ $=0$ ), equations (A1)-(A4) could be easily transformed into linear equations for $N_{A^{0}{ }_{1}}, N_{D^{+}{ }_{1}}$ and $N_{D^{0}}$, and into quadratic equations for $N_{A^{0}}, N_{D^{+}}$and $N_{D^{0}}{ }_{2}$. So, general expressions for $N_{A^{0}}, N_{D^{+}}$and $N_{D^{0}}$ values as well as approximate (at $b>>d, d>>b$ and $b \approx d$ ) expressions for $N_{A^{0}{ }_{2}}, N_{D^{+}}$and $N_{D^{0}}$ values appear as a result of solution of Eqs (A1)-(A4). The first ( $N_{A^{0}}$, $N_{D^{+}}$and $N_{D^{0}}{ }_{1}$ ) are given in the main text by the equations in (7). The latter $\left(N_{A^{0}}, N_{D^{+}{ }_{2}}\right.$ and $\left.N_{D^{0}}\right)$ are: 
K.D. Glinchuk, A.V. Prokhorovich: Analysis of the near-band-edge luminescence ...

$$
\begin{aligned}
& N_{A^{0} 2} \approx \frac{c_{p A}^{-} \delta p}{c_{p A}^{-} \delta p+c_{n A}^{0} \delta n+c_{n}^{*} \varphi_{D^{0}} N_{D 2}} N_{A 2}, \\
& N_{D^{+} 2} \approx\left(1-\varphi_{D^{0}}\right) N_{D 2}+\frac{\varphi_{D^{0}} c_{n}^{*} d}{a+c_{n}^{*} b} N_{D 2}, \\
& N_{D^{0} 2} \approx \varphi_{D^{0}} N_{D 2},
\end{aligned}
$$

if $b>>d\left\{\right.$ then $\left.\left[\varphi_{D^{0}} c_{n}^{*} d /\left(a+c_{n}^{*} b\right)\right] N_{D 2}<<N_{D 2}\right\}$, $N_{A^{0} 2} \approx \varphi_{A^{0}} N_{A 2}$,

$N_{D^{+} 2} \approx\left(1-\varphi_{D^{0}}\right) N_{D 2}+\frac{\varphi_{D^{0}} c_{n}^{*} d}{a+c_{n}^{*} d} N_{D 2}$,

$$
N_{D^{0} 2} \approx \frac{c_{n D}^{+} \delta n}{c_{n D}^{+} \delta n+c_{p D}^{0} \delta p+c_{n}^{*} \varphi_{A}{ }^{0} N_{A 2}} N_{D 2},
$$

if $d>>b$, and

$$
\begin{aligned}
& N_{A^{0} 2} \approx \frac{\sqrt{a\left(a+4 c_{n}^{*} d\right)}-a}{2 c_{n}^{*}\left(c_{p A}^{-} \delta p+c_{n A}^{0} \delta n\right)}, \\
& N_{D^{0} 2} \approx \frac{\sqrt{a\left(a+4 c_{n}^{*} b\right)}-a}{2 c_{n}^{*}\left(c_{n D}^{+} \delta n+c_{p D}^{0} \delta p\right)}, \\
& N_{D^{+} 2} \approx \\
& \approx \frac{a+2 c_{n}^{*}\left(c_{n D}^{+} \delta n+c_{p D}^{0} \delta p\right) N_{D 2}-\sqrt{a\left(a+4 c_{n}^{*} b\right)}}{2 c_{n}^{*}\left(c_{n D}^{+} \delta n+c_{p D}^{0} \delta p\right)},
\end{aligned}
$$

if $b \approx d\left(|b-d|<<a / c_{n}^{*}\right)$.

The $\varphi_{A^{0}}$ and $\varphi_{D^{0}}$ values (they are also given in the main text, see Sec. 2), could easily be found from Eqs (A1)-(A4).

\section{Bound and free exciton concentrations.}

Excitonic emission intensities $I_{A^{0} X}, I_{D^{+} X}$ and $I_{D}{ }^{0} X$

In this subsection expressions for $n_{A^{0} X 1,2}, n_{D^{+} X 1,2}$, $n_{D^{0} X 1,2}, n_{X}, I_{A^{0} X}, I_{D^{+} X}$ and $I_{D^{0} X}$ values, valid at any real relations between $n_{A^{0} X 1,2}$ and $N_{A^{0} 1,2}, n_{D^{+} X 1,2}$ and $N_{D^{+}{ }_{1,2}}, n_{D^{0} X 1,2}$ and $N_{D^{0}{ }_{1,2}} \quad\left(n_{A^{0} X 1,2} \leq N_{A^{0} 1,2}\right.$, $\left.n_{D^{+}{ }_{1,2}} \leq N_{D^{+} 1,2}, n_{D^{0}{ }_{1,2}} \leq N_{D^{0} 1,2}\right)$, will be given.

For the case discussed (see Sec. 2), as follows from the obvious steady-state rate equations for the concentrations of bound and free excitons

$$
b_{A^{0} X}\left(N_{A^{0} 1,2}-n_{A^{0} X 1,2}\right) n_{X}=\alpha_{A^{0} X} n_{A^{0} X 1,2},
$$

$$
\begin{aligned}
& b_{D^{+} X}\left(N_{D^{+} 1,2}-n_{D^{+} X 1,2}\right) n_{X}=\alpha_{D^{+} X} n_{D^{+} X 1,2}, \\
& b_{D^{0} X}\left(N_{D^{0} 1,2}-n_{D^{0} X 1,2}\right) n_{X}=\alpha_{D^{0} X} n_{D^{0} X 1,2},
\end{aligned}
$$

$b_{X} \delta p \delta n=$

$=\left[\alpha_{X}+b_{A^{0} X}\left(N_{A^{0}}-n_{A^{0} X}\right)+b_{D^{+} X}\left(N_{D^{+}}-n_{D^{+} X}\right)+\right.$

$\left.+b_{D^{0} X}\left(N_{D^{0}}-n_{D^{0} X}\right)\right] n_{X}$,

the $n_{A^{0} X 1,2}, n_{D^{+} X 1,2}, n_{D^{0} X 1,2}$ and $n_{X}$ stationary val-

$n_{A^{0} X 1,2}=\frac{b_{A^{0} X} n_{X}}{\alpha_{A^{0} X}+b_{A^{0} X} n_{X}} N_{A^{0} 1,2}$,

$n_{D^{+} X 1,2}=\frac{b_{D^{+} X} n_{X}}{\alpha_{D^{+} X}+b_{D^{+} X} n_{X}} N_{D^{+}{ }_{1,2}}$,

$n_{D^{0} X 1,2}=\frac{b_{D^{0} X} n_{X}}{\alpha_{D^{0} X}+b_{D^{0} X} n_{X}} N_{D^{0} 1,2}$,

$n_{X}=\frac{b_{X} \delta p \delta n}{\alpha_{X}+\beta b_{A^{0} X} N_{A^{0}}+\gamma b_{D^{+} X} N_{D^{+}}+z b_{D^{0} X} N_{D^{0}}}$.

Here $n_{A^{0} X}=n_{A^{0} X 1}+n_{A^{0} X 2}, n_{D^{+} X}=n_{D^{+} X 1}+n_{D^{+} X 2}$, $n_{D^{0} X}=n_{D^{0} X 1}+n_{D^{0} X 2}$ and $N_{A^{0}}=N_{A_{1}{ }_{1}}+N_{A^{0}{ }_{2}}$, $N_{D^{+}}=N_{D^{+}{ }_{1}}+N_{D^{+}{ }_{2}}, N_{D^{0}}=N_{D^{0}{ }_{1}}+N_{D^{0}{ }_{2}}$ are the total concentrations of bound excitons $A^{0} X, D^{+} X$, $D^{0} X$ and of neutral acceptors as well as ionized and neutral donors;

$\beta=\alpha_{A^{0} X} /\left(\alpha_{A^{0} X}+b_{A^{0} X} n_{X}\right)$,

$\gamma=\alpha_{D^{+} X} /\left(\alpha_{D^{+} X}+b_{D^{+} X_{X}} n_{X}\right)$,

$z=\alpha_{D^{0} X} /\left(\alpha_{D^{0} X}+b_{D^{0} X} n_{X}\right)$.

Obviously, $n_{X} \sim \delta p \delta n$ if

$\alpha_{X}+\beta b_{A^{0} X} N_{A^{0}}+\gamma b_{D^{+} X^{+}} N_{D^{+}}+z b_{D^{0} X} N_{D^{0}} \neq f(L)$,

i.e. at $\beta b_{A^{0} X} N_{A^{0}}+\gamma b_{D^{+} X} N_{D^{+}}+z b_{D^{0} X} N_{D^{0}} \neq f(L)$ or at $\alpha_{X}>\beta b_{A^{0} X} N_{A^{0}}+\gamma b_{D^{+} X} N_{D^{+}}+z b_{D^{0} X} N_{D^{0}}$, if

$\beta b_{A^{0} X} N_{A^{0}}+\gamma b_{D^{+} X} N_{D^{+}}+z b_{D^{0} X} N_{D^{0}}=f(L)$.

At assumptions made (see Sec. 2), the excitonic emission intensities $I_{A^{0} X}, I_{D^{+} X}$ and $I_{D^{0} X}$ obviously are given by the following relations:

$$
I_{A^{0} X}=\alpha_{A^{0} X} n_{A^{0} X}=\frac{\alpha_{A^{0} X}}{\alpha_{A^{0} X}+b_{A^{0} X} n_{X}} b_{A^{0} X} N_{A^{0}} n_{X},
$$




\section{K.D. Glinchuk, A.V. Prokhorovich: Analysis of the near-band-edge luminescence ...}

$$
\begin{aligned}
& I_{D^{+} X}=\alpha_{D^{+} X} n_{D^{+} X}= \\
& =\frac{\alpha_{D^{+} X}}{\alpha_{D^{+} X}+b_{D^{+} X} n_{X}} b_{D^{+} X} N_{D^{+}} n_{X} \\
& I_{D^{0} X}=\alpha_{D^{0} X^{0}{ }^{0}{ }^{0} X}=\frac{\alpha_{D^{0} X}}{\alpha_{D^{0} X}+b_{D^{0} X} n_{X}} b_{D^{0} X^{0}} N_{D^{0}} n_{X} .
\end{aligned}
$$

As one can see, at low $n_{X}$ values $\left(b_{A^{0} X} n_{X}<<\alpha_{A^{0} X}\right.$, $b_{D^{+} X} n_{X}<<\alpha_{A^{+} X}$ and $b_{D^{0} X} n_{X}<<\alpha_{D^{0} X}$, then, obviously, $n_{A^{0} X 1,2} \ll N_{A^{0} 1,2}, n_{D^{+} X 1,2} \ll N_{D^{+} 1,2}$, $n_{D^{0} X 1,2}<N_{D^{0} 1,2}, \beta \cong \gamma \cong \mathrm{Z} \cong 1$ and $I_{A^{0} X}, I_{D^{+} X}$, $\left.I_{D^{0} X} \sim n_{X}\right)$, Eqs (A19)-(A21) transform into Eqs (4)(6) that are used for the analysis of the excitonic emission intensities $I_{A^{0} X}, I_{D^{+} X}$ and $I_{D^{0} X}$ [in this case $n_{X} \sim$ $\delta p \delta n$ if $\alpha_{X}+b_{A^{0} X^{0}} N_{A^{0}}+b_{D^{+} X^{+}} N_{D^{+}}+b_{D^{0} X^{0}} N_{D^{0}} \neq f(L)$,

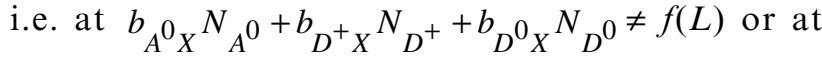
$\alpha_{X}>>b_{A^{0} X} N_{A^{0}}+b_{D^{+} X} N_{D^{+}}+b_{D^{0} X} N_{D^{0}}$, if $\left.b_{A^{0} X} N_{A^{0}}+b_{D^{+} X} N_{D^{+}}+b_{D^{0} X} N_{D^{0}}=f(L)\right]$. At high $n_{X}$

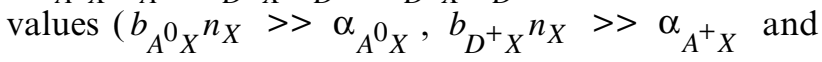
$b_{D^{0} X} n_{X}>>\alpha_{D^{0} X}$, then, obviously, $n_{A^{0} X 1,2} \cong N_{A^{0} 1,2}$, $\left.n_{D^{+} X 1,2} \cong N_{D^{+} 1,2}, n_{D^{0}{ }_{X 1,2}} \cong N_{D^{0}{ }_{1,2}}\right)$, the excitonic emission intensities $I_{A^{0} X} \cong \alpha_{A^{0} X^{N}}{ }_{A^{0}}, I_{D^{+} X} \cong$ $\cong \alpha_{D^{+} X} N_{D^{+}}$and $I_{D^{0} X} \cong \alpha_{D^{0} X} N_{D^{0}}$.

\section{References}

1. T.Taguchi, J.Shirafuji, Y.Inuishi. Excitonic emission in cadmium telluride // phys. stat.sol (b), 68 (2), pp.727-738 (1975)

2. A.A.Berg, P.J.Dean. Light-emitting diodes. Mir, Moskow (1979) (in Russian)

3. V.E.Lashkarev, A.V.Lubchenko, M.K.Sheinkman. Nonequilibrium process in photoconductors. Naukova Dumka, Kyiv (1981) (in Russian)

4. K.D.Glinchuk, A.V.Prokhorovich. Peculiarities of determination of the shallow-level impurity concentrations in semiconductors from the analysis of the exciton luminescence spectrum // Fiz.Technic.Poluprov. 36(5), pp.519-524 (2002) (in Russian)

5. T.Schmidt, K.Lischka, W.Zulehner. Excitation-power dependence of the near-band-edge photoluminescence in semiconductors // Phys.Rev.B.,45(16), pp.8989-8994 (1992)

6. K.D.Glinchuk, A.V.Prokhorovich. Peculiarities of determination of the shallow impurity concentrations in semiconductors from the analysis of the edge luminescence// Fiz. Technic. Poluprov. 37(2), pp. 159-165 (2003) (in Russian) 